\title{
Coronary risk factors in various occupational groups: The Oslo study
}

\author{
I. HOLME, A. HELGELAND, I. HJERMANN, P. LEREN, AND \\ P. G. LUND-LARSEN
}

From Life Insurance Companies' Institute for Medical Statistics in Oslo and Medical Outpatient Clinic, Ullevaal Hospital, Oslo, Norway

SUMMARY Coronary risk factors (CRF) have been recorded for nearly 15000 men aged between 40 and 49 living in Oslo in 1972. These data are matched with the 1970 census data for Norway so as to analyse CRF within various occupational groups. A fairly good agreement between mortality statistics for Norway and CRF was found in many occupations. For instance, persons in pedagogical work have low total mortality rates consistent with their low coronary risk factors. Taxi and busdrivers, on the other hand, have been reported to have mortality rates from lung cancer and coronary heart disease consistent with their high smoking prevalence, serum triglycerides, and cholesterol levels.

Norwegian mortality statistics show large differences in cause-specific and total mortality between various occupational groups (Central Bureau of Statistics of Norway, 1976). The reasons for these differences are not known, but to explain some of the variations in mortality from coronary heart disease (CHD), well established coronary risk factors (CRF) for CHD-such as, raised levels of serum cholesterol, high blood pressure, cigarette smoking-were recorded in men living in Oslo in 1972 aged between 40 and 49 years (Leren et al., 1975).

The main purpose of this study is to report levels of CRF within different occupational groups and discuss these values in the light of the mortality statistics given by the Central Bureau of Statistics of Norway (1976).

\section{Material and methods}

During the period from May 1972 to December 1973 all men in Oslo who were aged between 40 and 49 were asked to attend the Oslo Health Department for a mass $x$-ray examination at which measurements of height, weight, and blood pressure were taken. A non-fasting blood sample was also taken for analysis of serum cholesterol, triglycerides, and glucose. A questionnaire had been sent to them in advance, asking them to give details of previous diagnosed cardiovascular disease or diabetes, their physical activity at work and at leisure, smoking habits, etc. This questionnaire was thero matched with the other information. With consen? from the Alderman for Hospitals in Oslo and the Central Bureau of Statistics in Norway, information on each person's occupation from the 1970 census was added to the data file on condition that all EDP-procedures were done by the bureau and controlled in accordance with Norwegian law.

All occupations were coded in accordance with the Nordic classification of occupations. Although information can be obtained from the five digits of the code, details from only the first three digits were used.

The analysis was performed only on a symptomfree subgroup of those who attended as all persons with diagnosed cardiovascular disease or diabetes were excluded; this exclusion left 14677 men aged between 40 and 49 available for analysis. Further details are given in Leren et al. (1975).

A multiplicative risk score was computed based on previous prospective studies in Oslo (Natvig et al., 1968; Westlund and Nicolaysen, 1972), using the number of cigarettes smoked each day, serum cholesterol level, and systolic blood pressure as risk factors.

The CRF for a group was calculated as the arithmetic mean and differences between means were tested by a modified Student's $t$ test which took into account unequal variances between groups. 


\section{Results}

The Table presents a list of selected occupations with their CRF. Architects, engineers, chemists, and physicists all have a low-risk profile combined with a high proportion of attendance. Many have given up cigarette smoking and their lipid values, especially triglycerides, and blood pressure are lower than the mean values for all occupations.

Doctors have a very low attendance at screening $(23 \cdot 2 \%)$, making it difficult to draw conclusions. Those who did attend showed a marked low-risk profile of $4 \cdot 5$. Both doctors and dentists have about $15 \%$ lower triglycerides than the average.

Persons in pedagogical work have a marked low-risk profile. Between the various subgroups of pedagogical work there are some noteworthy differences in the risk factors. Lecturers in secondary school have a low smoking prevalence $(14.4 \%)$ compared with teachers at lower and higher educational levels $(24.8 \% \mathrm{P}<0.05)$. On the other hand, blood pressures in lecturers seem to be somewhat higher than in other groups of teachers and significantly higher than in headmasters $(P<0.05)$. There is also a tendency towards lower lipid values for those teachers who are most highly educated.

Persons in religious work generally smoke few cigarettes. Their lipid values and blood pressure are also below the mean values, resulting in a low risk score $(r=4 \cdot 1)$. However, their percentage of attendance was low $(47 \cdot 4 \%)$. Those in juridical work also have a low risk profile $(r=4.9)$, but again the attendance rate was low (52.9\%).

Leading executives in state administration, directors and managers in business, most of whom are well educated, have only a medium risk profile compared to the low risk for the above mentioned professional groups who also have higher education; a higher prevalence of smoking and raised serum cholesterol seem to be the two explanatory factors.

Persons in clerical and sales work have about the same risk score as the total population, but a higher proportion of them smoke than the average.

Ships' officers and pilots have about $50 \%$ excess risk score and a high percentage of them were cigarette smokers $(57 \cdot 4 \%)$. Their serum cholesterol level was $(278.8 \mathrm{mg} / \mathrm{dl})$ but few of them were able to attend $(34 \cdot 5 \%)$.

The coronary risk in road transport workers is high because of their high serum cholesterol level and the fact that many of them smoke. Taxidrivers seem to be the subgroup which has the highest risk score $(r=17 \cdot 0)$, and further analysis revealed a particularly high risk score for taxi and busdrivers with both a low income and poor education $(r=24 \cdot 0)$. These men are characterised by being both overweight and heavy cigarette smokers, and having high lipid values. Railway guards, on the other hand, have low lipid values and low blood pressure and have only a slightly raised smoking prevalence, giving a low risk score $(r=7 \cdot 1)$. However, despite a high attendance rate $(81.0 \%)$ only 30 persons in this group attended the screening. Bus and tramdrivers again have a higher risk score because of their high blood pressure and lipid values.

Metal foundry workers have a high risk score $(r=15 \cdot 4)$ because of high values in all three risk factors.

The large occupational groups of iron and metal workers and electricians have only a slight excess risk score caused by moderately raised lipid values and a high prevalence of cigarette smoking.

Some other workers-such as, operators in earthmoving, longshoremen, and vehicle loadersalso have a high risk profile as do the road transport workers. Waiters also have raised levels of cigarette smoking and serum cholesterol, but their attendance rate was only $41 \cdot 3 \%$.

\section{Discussion}

The process of selection to the various occupational groups may be associated with the observed CRF. The strength of this association is unknown, so care must be used in interpreting and discussing the results.

From data (not given here) it was shown that a socioeconomic selection took place regarding attendance at the screening. Low-status persons had a low attendance rate, while those of middle and upper middle class had the highest attendance rate. It may be that this selection in attendance has biased the results. An usual pattern in such surveys is that non-attenders have a higher risk profile than the attenders (Holme and Waaler, 1976). For instance, non-attenders have higher smoking prevalence than attenders. Thus, the observed high smoking prevalence in many low social status occupations is probably even higher because of the marked selection which took place.

It is known that Norwegian physicians have changed their smoking habits considerably during the last 20 years and the percentage of cigarette smokers in Oslo in 1974 was about $26 \%$ in the age group 35-54 years. The low smoking prevalence among physicians in our study $(17.7 \%)$ is probably due to an over-representation of internists and cardiologists in our small sample (23\%). Physicians have lower systolic blood pressure and 


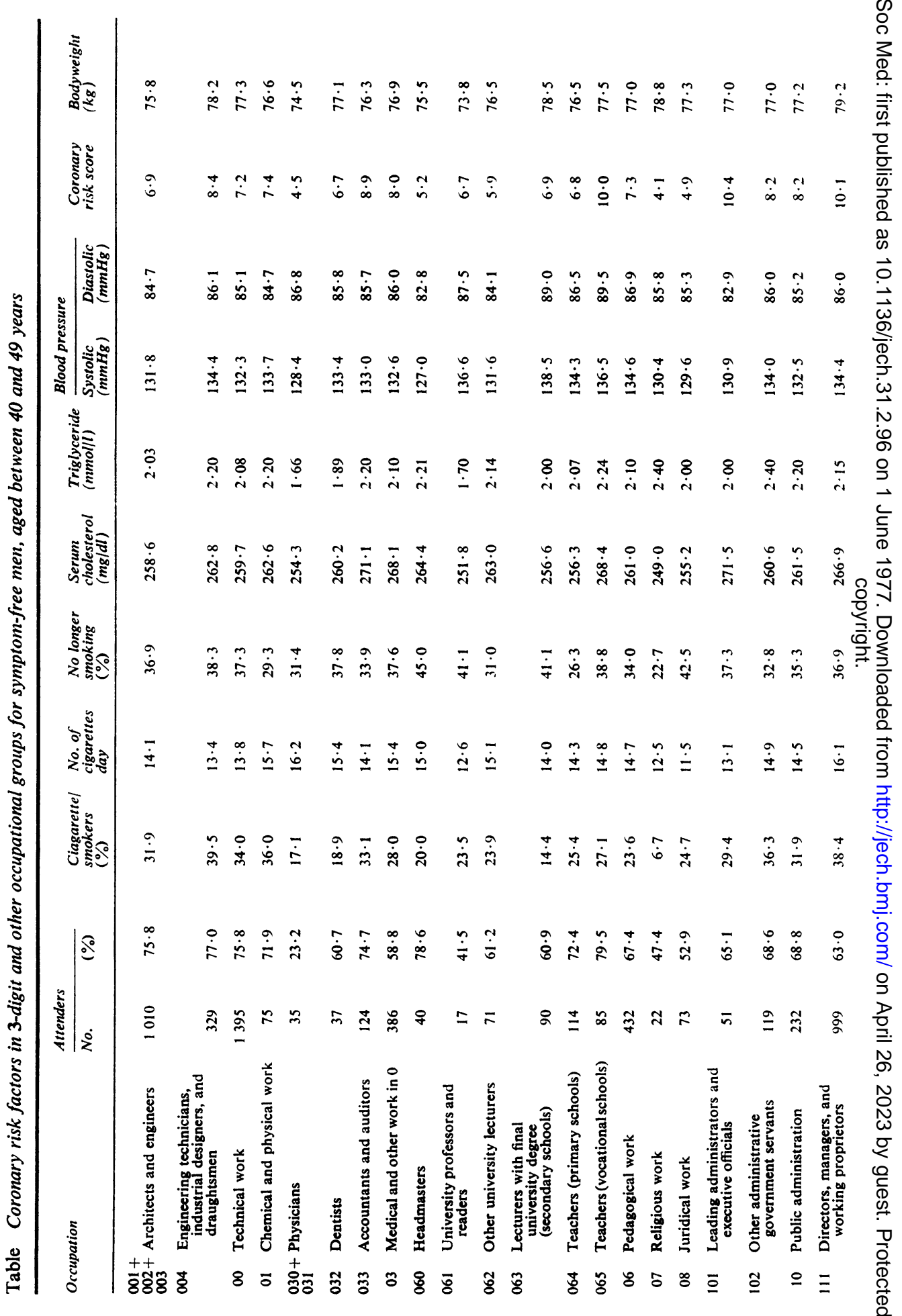




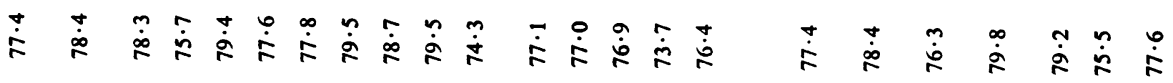

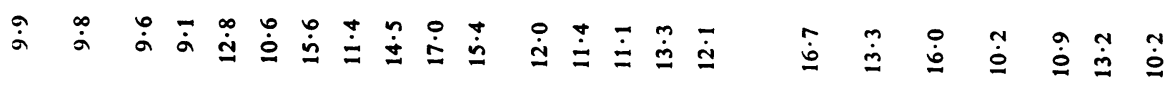

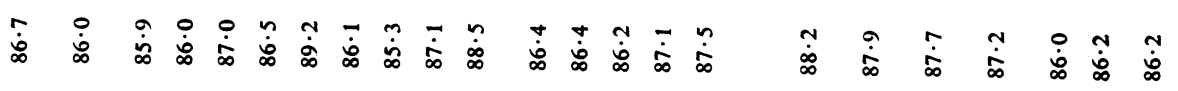

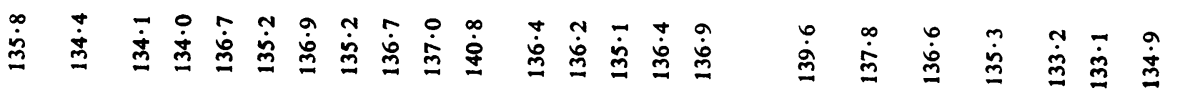

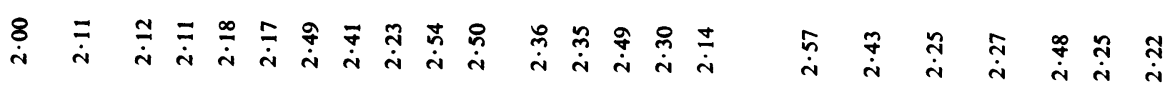

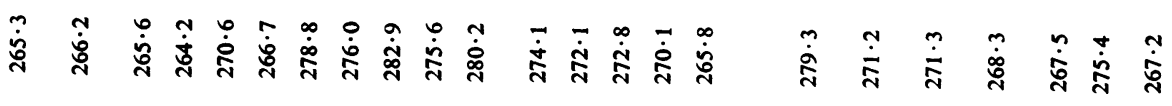

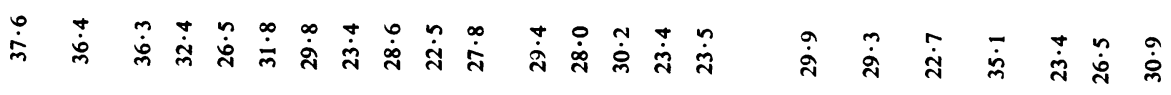

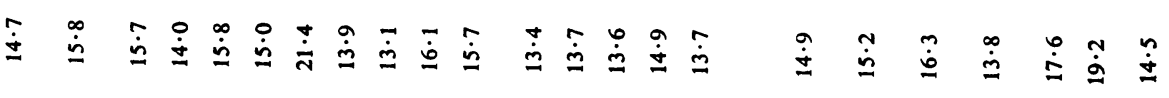

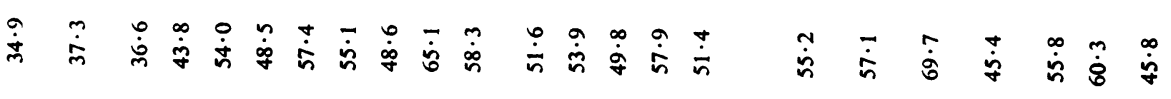

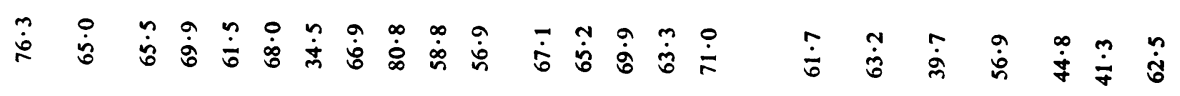

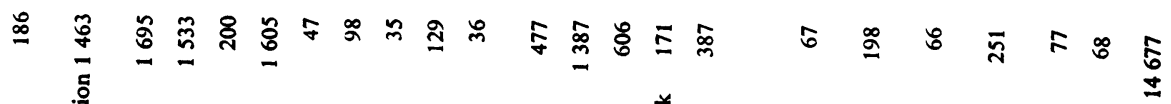

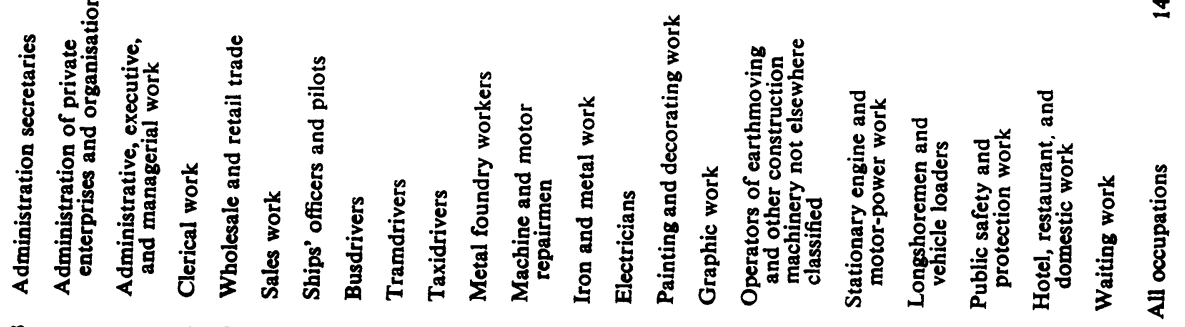

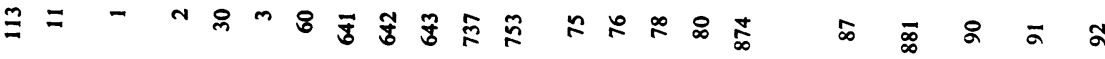


serum cholesterol levels than any other group of people and this relates to their mortality pattern (Holme, 1976). Their triglycerides are also very low. However, this may in part be explained by the probability that physicians who attended the screening clinic were more often fasting than were others.

People in other occupations who likewise require a high standard of education-such as, engineers, chemists, biologists, lawyers, dentists, priests-have a markedly low-risk profile and this again agrees with Norwegian mortality statistics of lung cancer, coronary heart disease, and total mortality (Central Bureau of Statistics of Norway, 1976).

A promising observation is the fact that teachers in secondary schools have stopped cigarette smoking to a large extent resulting in a very low cigarette smoking prevalence $(14 \cdot 4 \%)$. It is believed that these persons are opinion makers for young people so the argument of setting an example may be operating in explaining why these teachers smoke less than other categories of teachers. In total the pedagogical workers have a low risk profile in good agreement with their generally low mortality rates in Norway from lung cancer, coronary heart disease, and total mortality (Central Bureau of Statistics of Norway, 1976).

The occupational group, road transport workers, have a marked high risk profile. Almost all subgroups of drivers seem to smoke heavily and have high levels of triglycerides and serum cholesterol. Further analysis also revealed that taxi and busdrivers are far more sedentary at leisure than most people. An interesting contrast is the rather low risk profile in railway guards who probably are more active at work than drivers. Thus, these results support the observation of Morris et al. (1953) concerning busdrivers and conductors on London buses. However, the number of railway guards is low, so the results should be interpreted with caution. The high smoking prevalence in transport workers is in accordance with their high mortality from cancer of the lung. The group has also an excess mortality from CHD and sudden death consistent with our results (Central Bureau of Statistics of Norway, 1976).

Reprints from I. Holme, Life Insurance Companies' Institute for Medical Statistics in Oslo, Ullevaal Hospital, Oslo, Norway.

\section{References}

Central Bureau of Statistics of Norway (1976). Occupational Mortality 1970-1973. Statistical handbook 21. Central Bureau of Statistics: Oslo.

Holme, I. (1976). Mortality in Norwegian physicians in relation to smoking habits. Journal of the Norwegian Medical Association, 96, 620-627.

Holme, I., and Waaler, H. T. (1976). Five-year mortality in the city of Bergen, Norway, according to age, sex, \& and blood pressure. Acta medica Scandinavica, 200, 을 229-239.

Leren, P., Askevold, E. M., Foss, O. P., Frøili, A., $\vec{c}$ Grymyr, D., Helgeland, A., Hjermann, I., Holme, I., Lund-Larsen, P. G., and Norum, K. R. (1975). The Oslo study. Cardiovascular disease in middle-aged $\vec{\varphi}$ and young Oslo men. Acta medica Scandinavich $\exists$ Supplement, 588, 1-38.

Morris, J. N., Heady, J. A., Raffle, P. A. B., Robertsơ C. G., and Parks, J. W. (1953). Coronary heat disease and physical activity of work. Lancet, 2, 1053-1057.

Natvig, H., Borchgrevink, C. F., Dedichen, J., Owren, P. A. Schiøtz, E. H., and Westlund, K. (1968). A controlled trial of the effect of linolenic acid on incidence of coronary heart disease. The Norwegian vegetable oil experiment of 1965-1966. Scandinavian Journal of Clinical and Laboratory Investigation, 22, Supplement 105.

Westlund, K., and Nicolaysen, R. (1972). Ten year mortality and morbidity related to serum cholesterol. A follow-up of 3751 men aged 40-49. Scandinavian Journal of Clinical and Laboratory Investigation. 30, Supplement 127, 1-24. 